\title{
Backstepping Based Automatically Controlled Angiogenic Inhibition Therapy
}

\author{
R. Ozgur DORUK \\ Department of Electrical and Electronics Engineering \\ Atilim University, Incek, Golbasi, Ankara, TURKEY *
}

January 14,2020

\begin{abstract}
In this work, we present an automatically controlled angiogenic inhibition therapy where the variation profile of the inhibitory agent is generated by a control law that is derived using a back-stepping based control methodology. The angiogenic inhibition is described by a second order model representing the dynamics of tumor and supporting vasculature volumes. Backstepping control recursively stabilizes that dynamics and generates automatic control laws that allows the tumor volume to stay at a desired value. The desired value will be kept at one thousandth of its initial value. This will lead to a very small injection at the steady state. This is important as zero injection may lead to regrow of the tumor. The results will be presented in tabular and graphical forms. Tabular results present the variation of maximum injection rate and setup time. Graphical results present the variation of tumor and supporting vasculature volumes, injection rate and the tracking error between the reference and actual tumor volumes. In addition, we will also perform a simulation to test the capability of the closed loop to accomodate the parametric uncertainties in the rate constants. The uncertainties are represented by a random deviation in the range $\pm 10 \%$ times the nominal value of the effected parameter. The control laws will be kept the same and the simulations will be repeated by 1000 times and each result will be superimposed on the graph. The area occupied by the curves will show the relative capability of the designs.
\end{abstract}

\section{Angiogenic Inhibition Model of Cancer Progression}

In this research we will utilize the angiogenic inhibition model developed by [1] and 2. That is a nonlinear system represented by the following differential equations:

$$
\begin{aligned}
& \dot{x}_{1}=-\lambda_{1} x_{1} \ln \left(\frac{x_{1}}{x_{2}}\right) \\
& \dot{x}_{2}=b x_{1}-d x_{1}^{2 / 3} x_{2}-e x_{2} x_{3} \\
& \dot{x}_{3}=\int_{0}^{t} u(\tau) \exp \left(-\lambda_{3}(t-\tau)\right) d \tau
\end{aligned}
$$

where $x_{1}$ is the tumor volume in $\mathrm{mm}^{3}, x_{2}$ is the supporting vasculature volume in $\mathrm{mm}^{3}$ (blood vessels stem from a regular vessel due to tumor angiogenesis after secretion of vascular endothelial growth factor to the site), $x_{3}$ the level of inhibitory agent in the blood serum in $\mathrm{mg} / \mathrm{kg}$ and $u$ is the inhibitor administration rate in $\mathrm{mg} / \mathrm{kg}$.day. There are five parameters in (1) $\theta=\left[b, d, e, \lambda_{1}, \lambda_{3}\right]$ and their definitions, units and nominal values are available in Table 1 .

\footnotetext{
*e-mail: resat.doruk@atilim.edu.tr
} 
Table 1: The numerical values of parameters in (1). These are taken from [3] which presents a mathematical model of animal Lewis lung carcinoma.

\begin{tabular}{ccc}
\hline Parameter & Unit & Value \\
\hline$b$ & day $^{-1}$ & 5.85 \\
\hline$d$ & day $^{-1} \cdot \mathrm{mm}^{-2}$ & 0.00873 \\
\hline$e$ & day $^{-1} \cdot(\mathrm{mg} / \mathrm{kg})^{-2}$ & 0.66 \\
\hline$\lambda_{1}$ & day $^{-1}$ & 0.192 \\
\hline$\lambda_{3}$ & day $^{-1}$ & 1.3 \\
\hline
\end{tabular}

The integral equation found in the $\dot{x}_{3}$ equation is actually not very convenient for a control application. That is a convolution operation and defines the response of a linear system expressed by the following transfer function:

$$
H(s)=\frac{1}{s+\lambda_{3}}
$$

to the input $u(t)$. The value of the parameter $\lambda_{3}$ relatively closer to unity. Because of that the steady state gain of (2) is about 0.77 . This corresponds to a rise time of about 1.7 days. This seems quite fast concerning a realistic problem. So one can directly replace the plasma inhibitory agent variable $x_{3}$ by the inhibitory agent rate represented by $u$. This is also the case in the relevant examples presented by [4, 2. So one can reduce the third order model in (1) to a second order one by just replacing $x_{3}$ by $u$ in the $\dot{x}_{2}$ equation as:

$$
\begin{aligned}
& \dot{x}_{1}=-\lambda_{1} x_{1} \ln \left(\frac{x_{1}}{x_{2}}\right) \\
& \dot{x}_{2}=b x_{1}-d x_{1}^{2 / 3} x_{2}-e x_{2} u
\end{aligned}
$$

Uncontrolled evolution of the tumor volume and supporting vasculature volumes can be seen in Figure 1. The parameters are taken from Table 1. 

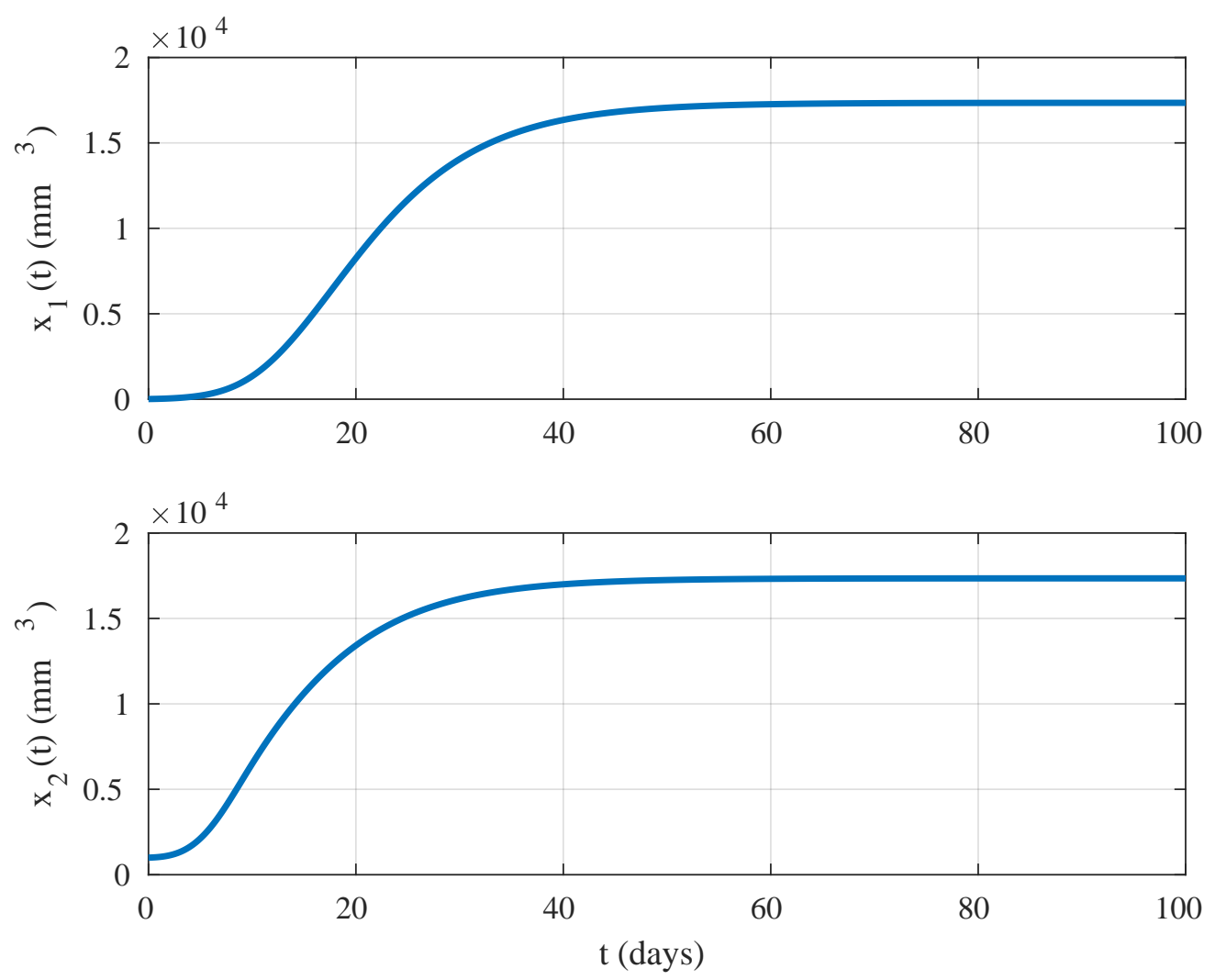

Figure 1: The evolution of tumor volume under no treatment conditions. The responses are obtained from (3) with the parameters in Table 1 .

\section{Application of Backstepping Control to Angiogenic Inhibition Problem}

In this section, we will apply the famous backstepping technique 5 to derive automatically controlled inhibitory agent injection laws. One should note here that, the angiogenic inhibition model presented in (3) is a full relative degree model. This means that one can allow the backstepping based approach without the risk of an uncontrollable remaining dynamics.

\subsection{Step 1: Stabilization of the Tumor Volume}

Before proceeding one needs to define a tracking error $\epsilon_{1}$ between actual and reference tumor volumes $\left(x_{1}\right.$ and $r_{1}$ ) as shown below:

$$
\epsilon_{1}=x_{1}-r_{1}
$$


Finding its dynamics by differentiating yields:

$$
\dot{\epsilon}_{1}=\dot{x}_{1}-\dot{r}_{1}=-\lambda_{1} x_{1} \ln \left(\frac{x_{1}}{x_{2}}\right)
$$

In the above we assume that the reference tumor level $r_{1}$ is constant. Although we are in Step 1, one needs to define the tracking error between the supporting vasculature volume and a virtual input $r_{2}$ as follows:

$$
\epsilon_{2}=x_{2}-r_{2}
$$

If one draws $x_{2}$ as $x_{2}=\epsilon_{2}+r_{2}$ and substitutes into (4), we can write the following:

$$
\dot{\epsilon}_{1}=-\lambda_{1} x_{1} \ln \left(\frac{x_{1}}{\epsilon_{2}+r_{2}}\right)
$$

To ensure the stability, one needs to define a Control Lyapunov Function $V_{1}$ as shown below:

$$
V_{1}=\frac{1}{2} \epsilon_{1}^{2}
$$

and taking its time derivative will yield:

$$
\dot{V}_{1}=\epsilon_{1} \dot{\epsilon}_{1}
$$

After substituting (7) to above, we will obtain:

$$
\dot{V}_{1}=\epsilon_{1}\left[-\lambda_{1} x_{1} \ln \left(\frac{x_{1}}{\epsilon_{2}+r_{2}}\right)\right]
$$

Concerning a standard backstepping application one needs to draw the virtual control variable $r_{2}$ from the above equation which will yield a negative definite $\dot{V}_{1}$. This will yield an asymptotically stable dynamics for $\epsilon_{1}$. One thing to note here is that backstepping techniques develop the control laws recursively. This recursiveness is provided by the Control Lyapunov Functions. In this step, this is obtained by equating (10) to a form shown by:

$$
\dot{V}_{1}=-\epsilon_{2} \epsilon_{1}-K_{1} \epsilon_{1}^{2}
$$

with $K_{1}$ being a positive real number. In order to obtain this rate function, $r_{2}$ should be derived as:

$$
r_{2}=\lambda_{1} x_{1} \ln \left(\frac{x_{2}}{x_{1}}\right)+x_{2}+K_{1} \epsilon_{1}
$$

\subsection{Step 2: Stabilization of the Supporting Vasculature Volume}

One should start by taking the time derivative of the tracking error between $x_{2}$ and $r_{2}$ as shown in the following:

$$
\dot{\epsilon}_{2}=-\lambda_{1}^{2} x_{1} \ln ^{2}\left[\frac{x_{1}}{x_{2}}\right]-\frac{\lambda_{1} b x_{1}^{2}}{x_{2}}+\lambda_{1} d x_{1}^{5 / 3}-\lambda_{1} e x_{1} u-\lambda_{1}^{2} x_{1} \ln \left[\frac{x_{1}}{x_{2}}\right]+K_{1} \lambda_{1} x_{1} \ln \left[\frac{x_{1}}{x_{2}}\right]
$$

Secondly, we will need to define a Control Lyapunov Function for this step. This should be defined as an additive to that of the first one.

$$
V_{2}=V_{1}+\frac{1}{2} \epsilon_{2}^{2}
$$

and its rate of change is:

$$
\dot{V}_{2}=\dot{V}_{1}+\epsilon_{2} \dot{\epsilon}_{2}=-K_{1} \epsilon_{1}^{2}-\epsilon_{2} \epsilon_{1}+\epsilon_{2} \dot{\epsilon}_{2}
$$

Substituting from (13) yields:

$$
\dot{V}_{2}=\epsilon_{2}\left[-\epsilon_{1}-\lambda_{1}^{2} x_{1} \ln ^{2}\left[\frac{x_{1}}{x_{2}}\right]-\frac{\lambda_{1} b x_{1}^{2}}{x_{2}}+\lambda_{1} d x_{1}^{5 / 3}-\lambda_{1} e x_{1} u-\lambda_{1}^{2} x_{1} \ln \left[\frac{x_{1}}{x_{2}}\right]+K_{1} \lambda_{1} x_{1} \ln \left[\frac{x_{1}}{x_{2}}\right]\right]
$$


If we choose an inhibitory agent injection rate as:

$$
u=\frac{-K_{2} \epsilon_{2}+\epsilon_{1}+\lambda_{1}^{2} x_{1} \ln ^{2}\left[\frac{x_{1}}{x_{2}}\right]+\frac{\lambda_{1} b x_{1}^{2}}{x_{2}}-\lambda_{1} d x_{1}^{5 / 3}+\lambda_{1}^{2} x_{1} \ln \left[\frac{x_{1}}{x_{2}}\right]-K_{1} \lambda_{1} x_{1} \ln \left[\frac{x_{1}}{x_{2}}\right]}{\lambda_{1} e x_{1}}
$$

(16) becomes:

$$
V_{2}=-K_{1} \epsilon_{2}^{2}-K_{2} \epsilon_{2}^{2}
$$

with $K_{2}$ being a positive real number. (18) ensures the asymptotic stability of the closed loop.

\section{Results}

In this section we will present an example application together with its simulation results. First of all, we will present a scenario for the simulation environment and secondly we will present the results for different controller configurations.

\subsection{Information on Simulations}

One can see the information related to the initial tumor and supporting vasculature volumes and targeted treatment duration in days in Table 2.

Table 2: Problem scenario and simulation conditions

\begin{tabular}{cccc}
\hline Scenario Parameter & Symbol & Value & Unit \\
\hline Treatment Duration & $T_{f}$ & 50 & Days \\
Initial Tumor Volume & $x_{1}(0)$ & 2000 & $\mathrm{~mm}^{3}$ \\
Initial Vasculature Volume & $x_{2}(0)$ & 250 & $\mathrm{~mm}^{3}$ \\
Reference (steady state) tumor volume & $r_{1}$ & $\frac{1}{1000} x_{1}(0)$ & $\mathrm{mm}^{3}$ \\
\hline
\end{tabular}

The purpose of the controlled simulations is to reduce the tumor volume $x_{1}$ to $1 \%$ of its initial volume $x_{1}(0)$ in Table 2 in a finite duration. The duration required to achieve this goal is called as setup time. In the control application there is a constant reference tumor volume $r_{1}$. This will be chosen as one thousandth of the initial volume i.e. $r_{1}=2.5 \mathrm{~mm}^{3}$.

\subsection{Simulation Results: No Uncertainties Exist}

A typical variation of the tumor volume $x_{1}(t)$, supporting vasculature volume $x_{2}(t)$ and injection rate of inhibitory agent $u(t)$ can be seen in Figure 2 

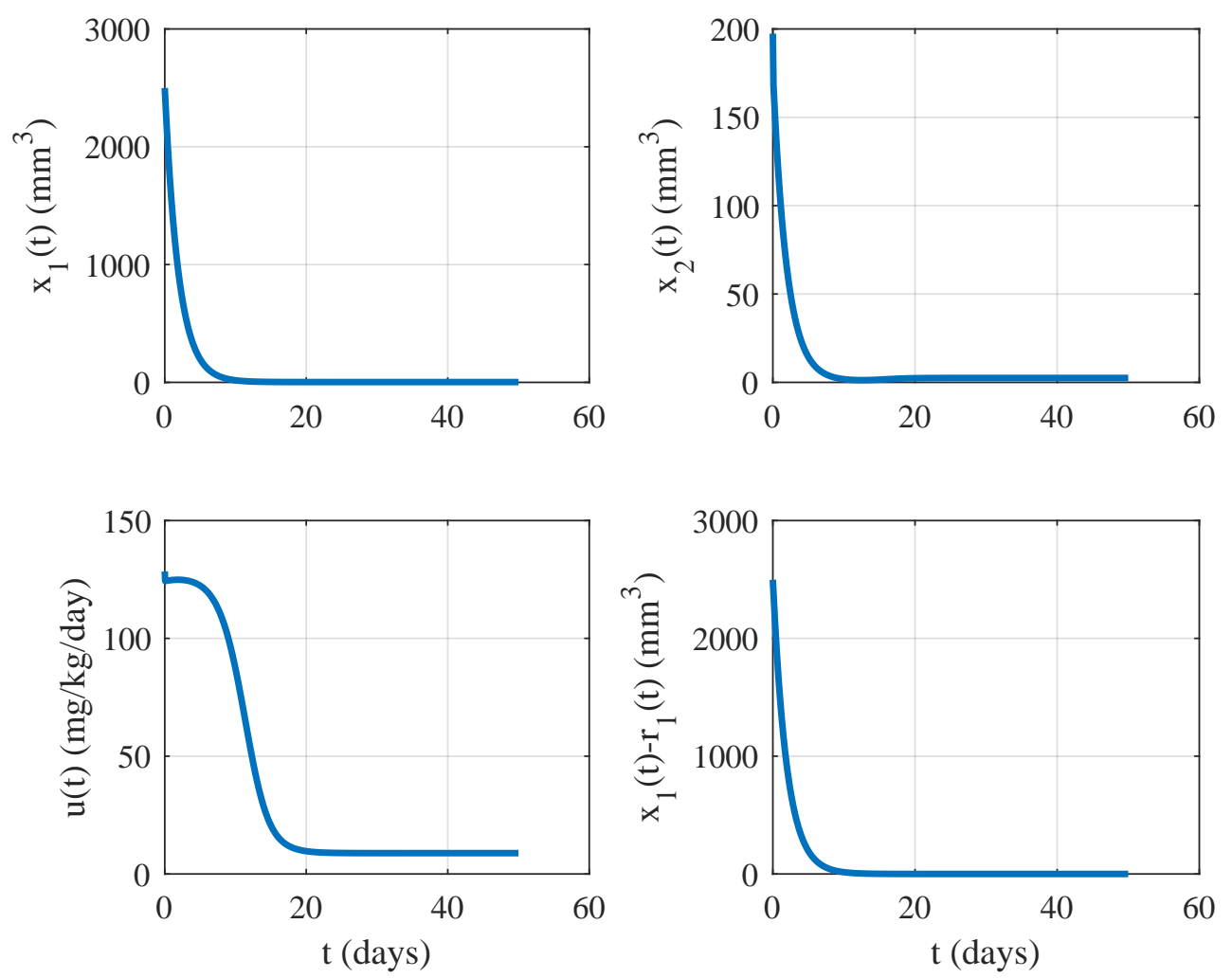

Figure 2: The variation of tumor volume $x_{1}(t)$, supporting vasculature volume $x_{2}(t)$ and injection rate of inhibitory agent $u(t)$ when $K_{1}=100$ and $K_{2}=0.5$.

In addition to 2, one is eligible to see the results associated with maximum required injection and setup times in Table 3. Note that some rows in this table depicts pretty unapplicable cases (i.e. instability or very large injection rates). 
bioRxiv preprint doi: https://doi.org/10.1101/2020.01.13.905067; this version posted January 14,2020 . The copyright holder for this preprint (which was not certified by peer review) is the author/funder, who has granted bioRxiv a license to display the preprint in perpetuity. It is made available under aCC-BY-NC-ND 4.0 International license.

Table 3: Maximum value of inhibitory injection rate and setup times of backstepping based treatment procedures. Here $\max (u)$ is the maximum inhibitory agent injection rate and $T_{s}$ is the setup time in days.

\begin{tabular}{|c|c|c|c|}
\hline$K_{1}$ & $K_{2}$ & $\max (u)$ & $T_{s}$ \\
\hline 2.0000 & 0.0500 & 1249.3485 & 5.5320 \\
\hline 2.0000 & 0.1000 & 2789.3545 & 5.0430 \\
\hline 2.0000 & 0.2000 & 44372.6944 & 4.3100 \\
\hline 2.0000 & 0.2500 & 4033991.5434 & 4.0360 \\
\hline 5.0000 & 0.0500 & 101.4783 & 17.8450 \\
\hline 5.0000 & 0.1000 & 103.2578 & 14.9000 \\
\hline 5.0000 & 0.2000 & 106.8169 & 11.2200 \\
\hline 5.0000 & 0.5000 & 400.8060 & 6.4910 \\
\hline 5.0000 & 0.9000 & 3584.5894 & 4.2040 \\
\hline 5.0000 & 1.5000 & 95733.1915 & 2.8020 \\
\hline 5.0000 & 2.5000 & 19222732.9454 & 1.8790 \\
\hline 5.0000 & 5.0000 & 137296569206.2600 & 1.2380 \\
\hline 10.0000 & 0.0100 & 81.3145 & 42.0260 \\
\hline 10.0000 & 0.0200 & 82.0646 & 38.5310 \\
\hline 10.0000 & 0.0500 & 84.3149 & 30.8440 \\
\hline 10.0000 & 0.1000 & 88.0653 & 23.1570 \\
\hline 10.0000 & 0.2000 & 95.5661 & 15.4700 \\
\hline 10.0000 & 0.5000 & 206.7063 & 7.7840 \\
\hline 10.0000 & 1.0000 & 2866.5315 & 4.2920 \\
\hline 10.0000 & 2.0000 & 538543.5230 & 2.3000 \\
\hline 20.0000 & 0.0002 & 42.3266 & 93.1330 \\
\hline 20.0000 & 0.0020 & 42.6035 & 89.9110 \\
\hline 20.0000 & 0.0100 & 43.8343 & 77.9290 \\
\hline 20.0000 & 0.0200 & 45.3727 & 66.8030 \\
\hline 20.0000 & 0.0500 & 49.9880 & 46.7770 \\
\hline 20.0000 & 0.1000 & 57.6802 & 31.2000 \\
\hline 20.0000 & 0.2000 & 73.0645 & 18.7390 \\
\hline 20.0000 & 0.5000 & 155.0376 & 8.5440 \\
\hline 20.0000 & 1.0000 & 2113.3121 & 4.4980 \\
\hline 20.0000 & 2.0000 & 385414.3820 & 2.3270 \\
\hline 20.0000 & 4.0000 & 12287655310.0452 & 1.2020 \\
\hline 50.0000 & 0.0020 & 9.7487 & 213.5450 \\
\hline 50.0000 & 0.0100 & 10.1500 & 156.6040 \\
\hline 50.0000 & 0.0200 & 10.6819 & 117.4580 \\
\hline 50.0000 & 0.0500 & 12.4766 & 67.1270 \\
\hline 50.0000 & 0.1000 & 16.2033 & 39.1650 \\
\hline 50.0000 & 0.2000 & 27.3737 & 21.3710 \\
\hline 50.0000 & 0.5000 & 131.6975 & 9.0520 \\
\hline 50.0000 & 1.0000 & 1789.5742 & 4.6230 \\
\hline 50.0000 & 2.0000 & 325013.6384 & 2.3420 \\
\hline 50.0000 & 4.0000 & 10618811896.1328 & 1.1810 \\
\hline 100.0000 & 0.0020 & 9.2806 & 391.9980 \\
\hline 100.0000 & 0.0100 & 9.6517 & 235.2020 \\
\hline 100.0000 & 0.0200 & 10.1496 & 156.8040 \\
\hline 100.0000 & 0.0500 & 11.8434 & 78.4060 \\
\hline 100.0000 & 0.1000 & 15.3737 & 42.7710 \\
\hline 100.0000 & 0.5000 & 128.4096 & 9.2310 \\
\hline 100.0000 & 1.0000 & 1696.9747 & 4.6640 \\
\hline 100.0000 & 2.0000 & 308350.3896 & 2.3460 \\
\hline 100.0000 & 2.2500 & 1131789.4217 & 2.0860 \\
\hline & & & \\
\hline
\end{tabular}




\subsection{Simulation Results: Simulations Under Existence of Uncertainties}

In this section, we will present simulations under the existence of $10 \%$ uncertainty in the rate constants (i.e. $\left.\left[b, d, e, \lambda_{1}\right]\right)$. The uncertainties are incormporated into the parameters by introducing random deviations to those parameters. This deviation is obtained by adding a uniformly distributed random number in the following fashion:

For example for parameter $b$, the uncertainty is incorporated as $\hat{b}=b(1+\delta)$ where $\delta$ is a random number uniformly distributed in the interval $[-0,1,0,1]$. This means that there is a $10 \%$ uncertainty assumed in the parameters. The deviated parameters are only used in the simulation of the angiogenic inhibition model itself. The controller stays same. In other words, controller's design involves the nominal values of the parameters $\theta=\left[b, d, e, \lambda_{1}\right]$.

Since we have randomly varying parameters, the simulation needs to be repeated. We repeat the simulations 1000 times and superimpose the results on the same figure. The hold on/off feature of the MATLAB environment draws each curve with a different color. As the number of runs advances, the curves may occupy a larger space. The larger the occupied area the larger the deviations.

The simulations are performed with the configurations presented in Table 4. If the results are not therapeutically feasible under $10 \%$ uncertainty, the situation is also indicated. The results associated with the therapeutically feasible cases are presented in Figures $\mathbf{3}, \mathbf{1 6}$ referenced in the table. It should be noted that, unstable and cases resulting negative inhibitory agent injections are not reported graphically.

Table 4: The configuration of the sliding surface parameters for the simulations under $10 \%$ uncertainty in the physiological rate constant parameters $\theta=\left[b, d, e, \lambda_{1}\right]$. If the results are not therapeutically feasible under $10 \%$ uncertainty, the situation is indicated in the observation column. NotE: When the gains are swapped (i.e. $\left.K_{1} \Leftrightarrow K_{2}\right)$ similar results are obtained.

\begin{tabular}{|c|c|c|}
\hline$K_{1}$ & $K_{2}$ & Observations \\
\hline 1 & 0.05 & UNSTABLE \\
\hline 1 & 0.02 & UNSTABLE \\
\hline 1 & 0.1 & UNSTABLE \\
\hline 0.5 & 0.02 & UNSTABLE \\
\hline 0.5 & 0.05 & UNSTABLE \\
\hline 2 & 0.1 & UNSTABLE \\
\hline 2 & 0.05 & UNSTABLE \\
\hline 2 & 0.2 & UNSTABLE \\
\hline 2 & 0.4 & UNSTABLE \\
\hline 5 & 0.1 & UNSTABLE \\
\hline 5 & 0.2 & UNSTABLE \\
\hline 5 & 0.5 & UNSTABLE \\
\hline 5 & 0.9 & UNSTABLE \\
\hline 10 & 0.01 & $\begin{array}{l}\text { Sudden decrease in the inhibitory agent administration rate } u(t) \text {. } \\
\text { Sudden jumps in the vasculature volume } x_{2}(t)\end{array}$ \\
\hline 10 & 0.05 & $\begin{array}{l}\text { Sudden decrease in the inhibitory agent administration rate } u(t) \text {. } \\
\text { Sudden jumps in the vasculature volume } x_{2}(t)\end{array}$ \\
\hline 10 & 0.1 & $\begin{array}{l}\text { Sudden decrease in the inhibitory agent administration rate } u(t) \text {. } \\
\text { Sudden jumps in the vasculature volume } x_{2}(t)\end{array}$ \\
\hline 10 & 0.2 & UNSTABLE \\
\hline 10 & 0.5 & UNSTABLE \\
\hline 10 & 1 & UNSTABLE \\
\hline 10 & 2 & UNSTABLE \\
\hline 20 & 0.0002 & $\begin{array}{l}\text { Sudden decrease in the inhibitory agent administration rate } u(t) \text {. } \\
\text { Sudden jumps in the vasculature volume } x_{2}(t) \\
\text { Very slow response }\end{array}$ \\
\hline
\end{tabular}




\begin{tabular}{|c|c|c|}
\hline 20 & 0.002 & $\begin{array}{c}\text { Sudden decrease in the inhibitory agent administration rate } u(t) \text {. } \\
\text { Sudden jumps in the vasculature volume } x_{2}(t) \\
\text { Very slow response }\end{array}$ \\
\hline 20 & 0.01 & $\begin{array}{c}\text { Sudden decrease in the inhibitory agent administration rate } u(t) \text {. } \\
\text { Sudden jumps in the vasculature volume } x_{2}(t) \\
\text { Very slow response }\end{array}$ \\
\hline 20 & 0.02 & $\begin{array}{c}\text { Sudden decrease in the inhibitory agent administration rate } u(t) \text {. } \\
\text { Sudden jumps in the vasculature volume } x_{2}(t) \\
\text { Very slow response }\end{array}$ \\
\hline 20 & 0.05 & $\begin{array}{c}\text { Sudden decrease in the inhibitory agent administration rate } u(t) \text {. } \\
\text { Sudden jumps in the vasculature volume } x_{2}(t)\end{array}$ \\
\hline 20 & 0.1 & $\begin{array}{l}\text { Sudden decrease in the inhibitory agent administration rate } u(t) \text {. } \\
\text { Sudden jumps in the vasculature volume } x_{2}(t)\end{array}$ \\
\hline 20 & 0.2 & $\begin{array}{l}\text { Sudden decrease in the inhibitory agent administration rate } u(t) \text {. } \\
\text { Sudden jumps in the vasculature volume } x_{2}(t)\end{array}$ \\
\hline 20 & 0.5 & UNSTABLE \\
\hline 20 & 1 & UNSTABLE \\
\hline 20 & 2 & UNSTABLE \\
\hline 20 & 4 & UNSTABLE \\
\hline 50 & 0.002 & NEGATIVE INJECTION \\
\hline 50 & 0.01 & NEGATIVE INJECTION \\
\hline 50 & 0.02 & NEGATIVE INJECTION \\
\hline 50 & 0.05 & NEGATIVE INJECTION \\
\hline 50 & 0.1 & NEGATIVE INJECTION \\
\hline 50 & 0.2 & $\begin{array}{c}\text { Sudden decrease in the inhibitory agent administration rate } u(t) \text {. } \\
\text { Sudden jumps in the vasculature volume } x_{2}(t) \\
\text { Relatively faster. }\end{array}$ \\
\hline 50 & 0.5 & $\begin{array}{l}\text { Sudden decrease in the inhibitory agent administration rate } u(t) \text {. } \\
\text { Sudden jumps in the vasculature volume } x_{2}(t) \\
\text { Smoother responses }\end{array}$ \\
\hline 50 & 1 & UNSTABLE \\
\hline 50 & 2 & UNSTABLE \\
\hline 50 & 5 & UNSTABLE \\
\hline 100 & 0.002 & NEGATIVE INJECTION \\
\hline 100 & 0.01 & NEGATIVE INJECTION \\
\hline 100 & 0.02 & NEGATIVE INJECTION \\
\hline 100 & 0.05 & NEGATIVE INJECTION \\
\hline 100 & 0.1 & NEGATIVE INJECTION \\
\hline 100 & 0.5 & Fast and smoother responses, no jumps. \\
\hline 100 & 1 & UNSTABLE \\
\hline 100 & 2 & UNSTABLE \\
\hline 100 & 5 & UNSTABLE \\
\hline 100 & 10 & UNSTABLE \\
\hline 200 & 0.2 & NEGATIVE INJECTION \\
\hline 200 & 0.5 & $\begin{array}{l}\text { Fast and smoother responses, no jumps. } \\
\text { Relatively larger injection rate. }\end{array}$ \\
\hline 200 & 1 & UNSTABLE \\
\hline 200 & 2 & UNSTABLE \\
\hline 200 & 5 & UNSTABLE \\
\hline 200 & 10 & UNSTABLE \\
\hline 500 & 0.2 & NEGATIVE INJECTION \\
\hline 500 & 0.5 & $\begin{array}{l}\text { Fast responses, jumps reappear. } \\
\text { Relatively larger injection rate. }\end{array}$ \\
\hline 500 & 1 & NEGATIVE INJECTION \\
\hline
\end{tabular}




\begin{tabular}{lll}
\hline 500 & 2 & UNSTABLE \\
\hline 500 & 5 & UNSTABLE \\
\hline
\end{tabular}
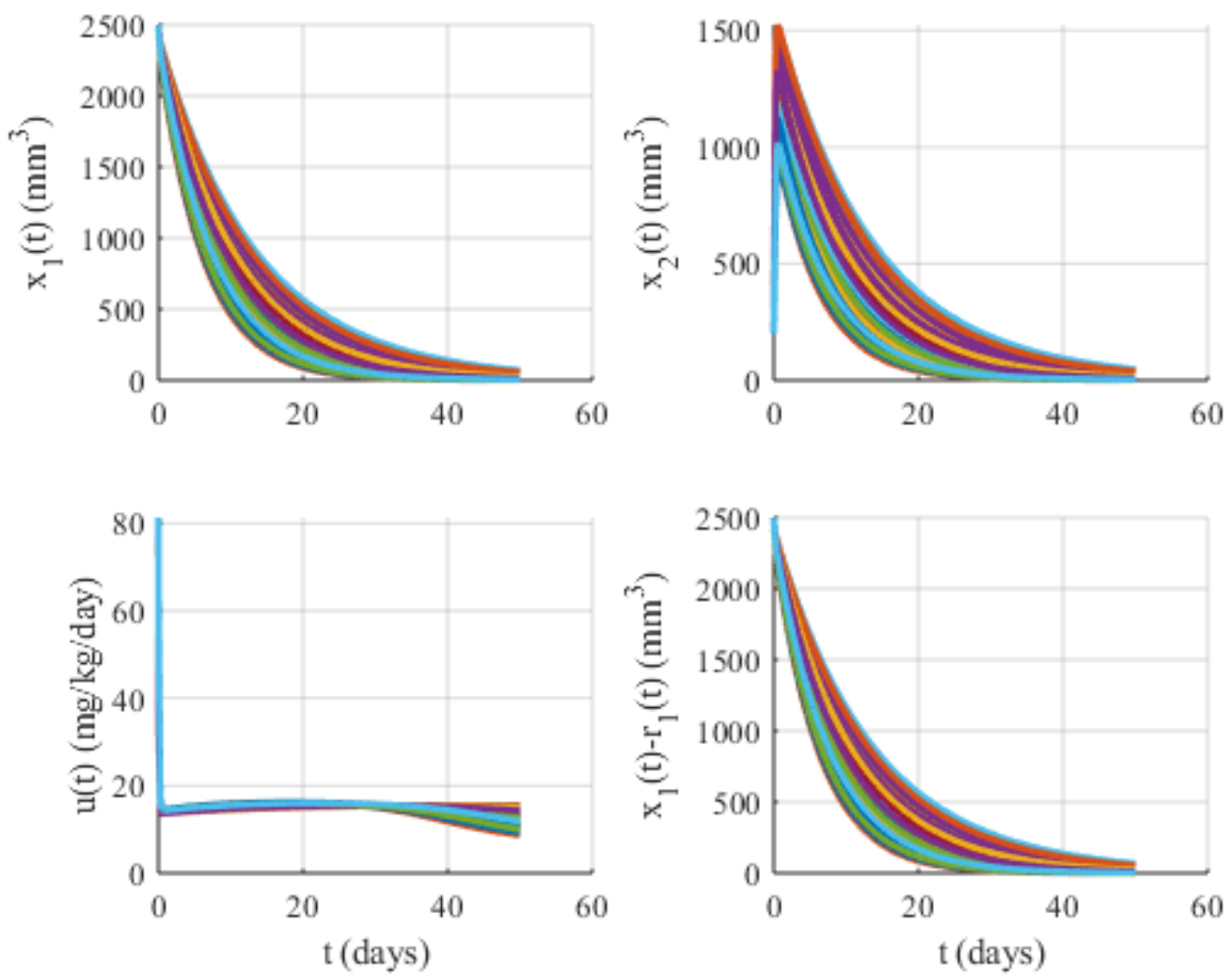

Figure 3: Simulations under the existence of $10 \%$ uncertainty when $K_{1}=10$ and $K_{2}=0.01 . x_{1}(t)$ : Tumor volume, $x_{2}(t)$ : Supporting vasculature volume, $u(t)$ : Inhibitory agent rate 

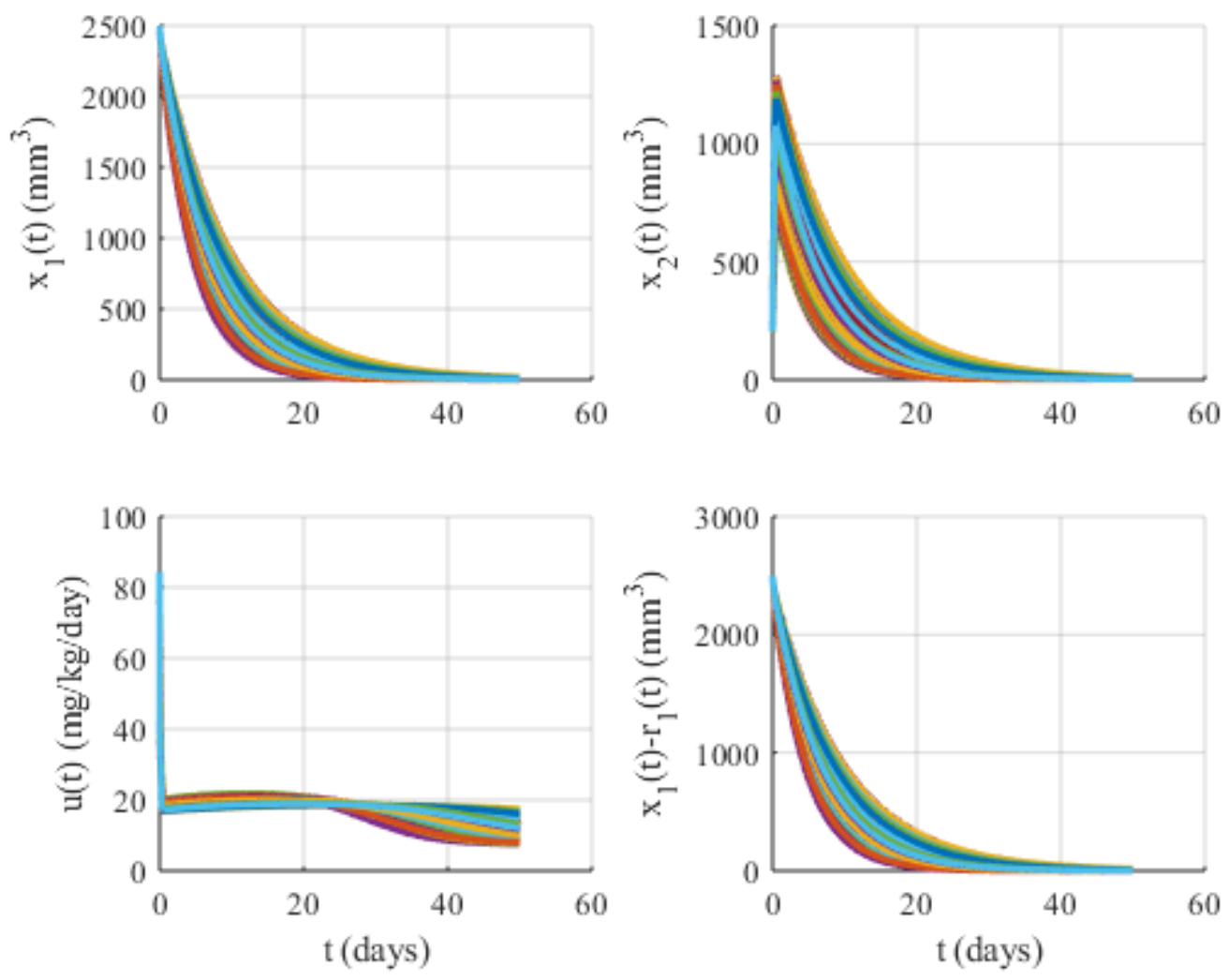

Figure 4: Simulations under the existence of $10 \%$ uncertainty when $K_{1}=10$ and $K_{2}=0.05 . x_{1}(t)$ : Tumor volume, $x_{2}(t)$ : Supporting vasculature volume, $u(t)$ : Inhibitory agent rate 

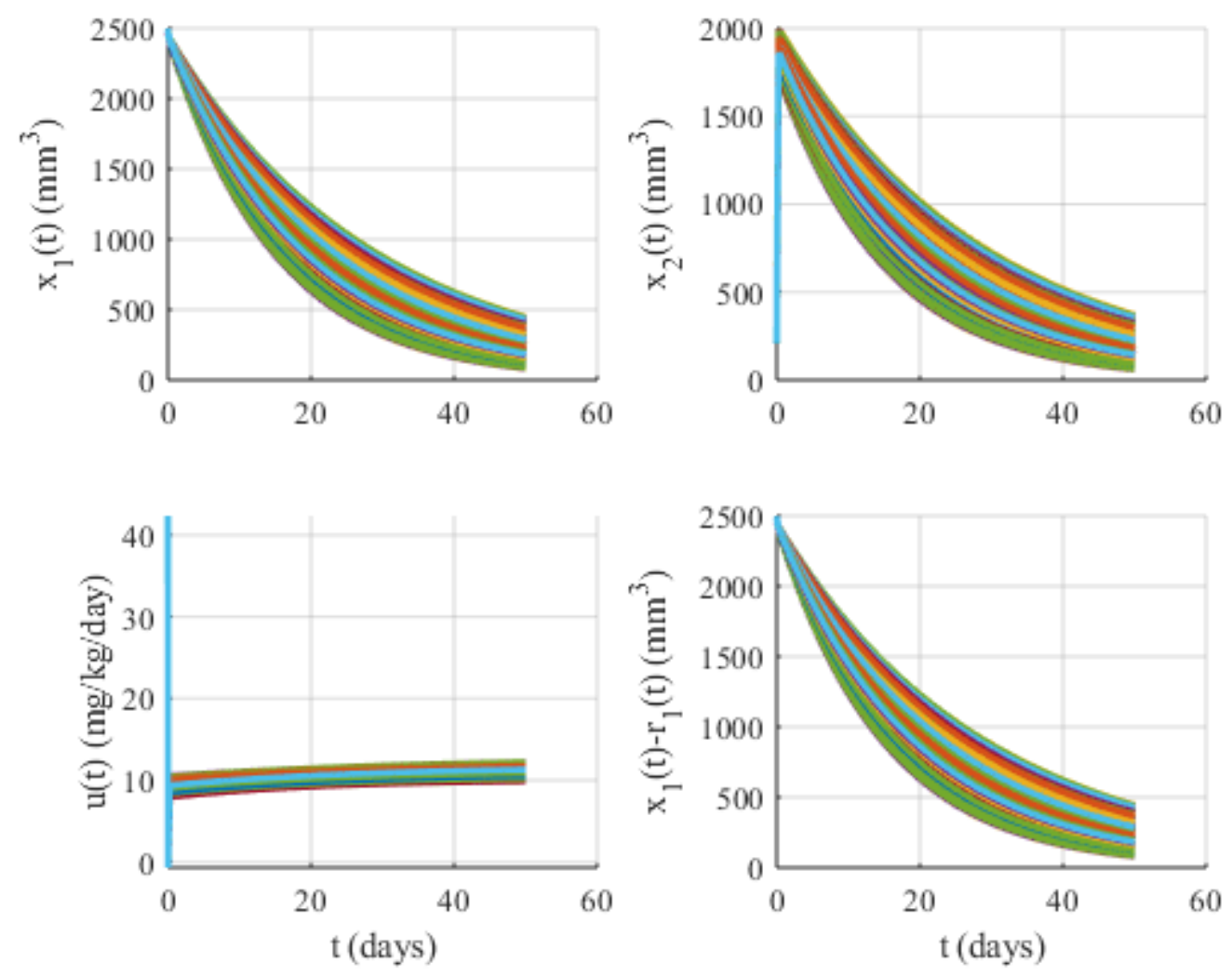

Figure 5: Simulations under the existence of $10 \%$ uncertainty when $K_{1}=20$ and $K_{2}=0.0002 . x_{1}(t)$ : Tumor volume, $x_{2}(t)$ : Supporting vasculature volume, $u(t)$ : Inhibitory agent rate 

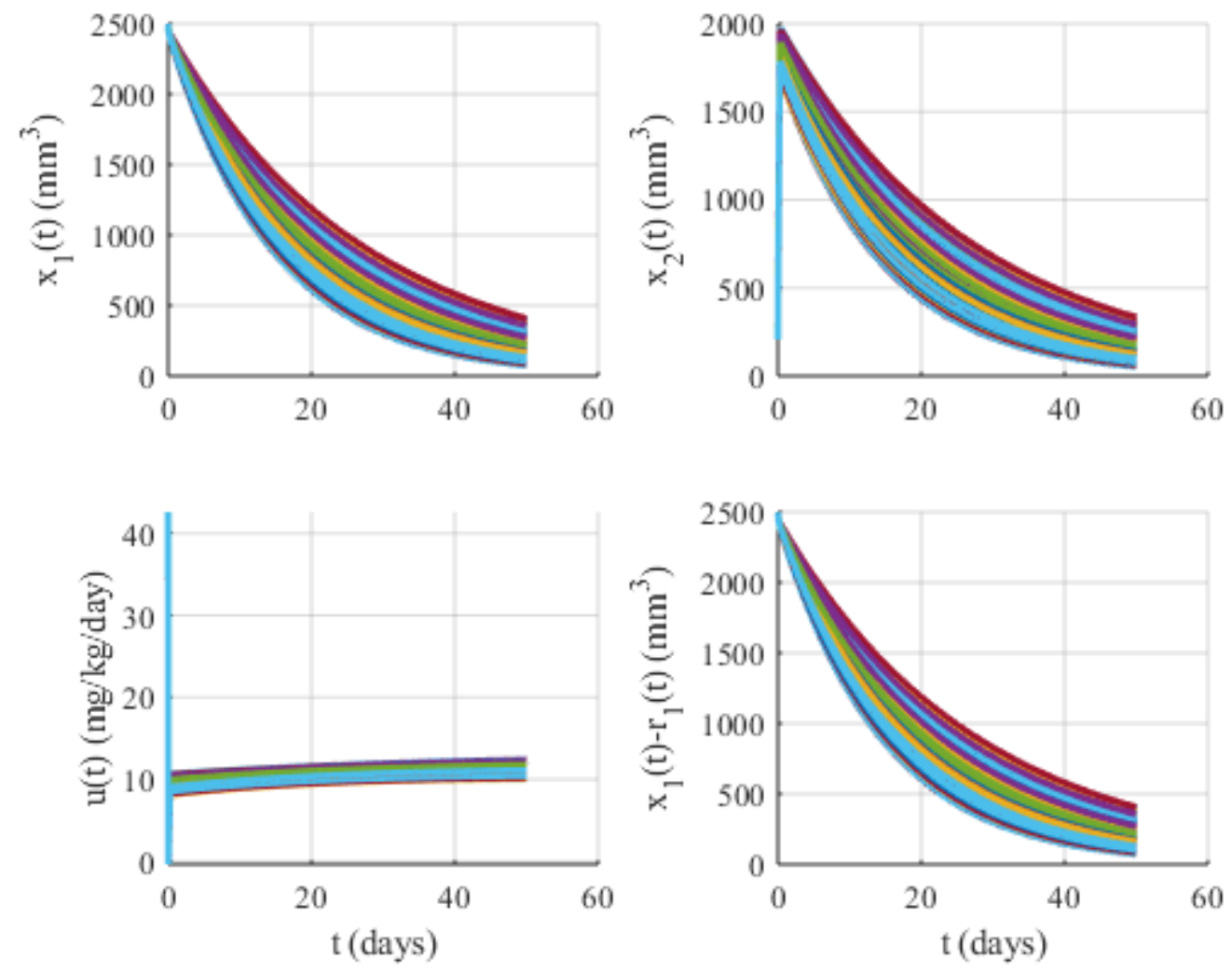

Figure 6: Simulations under the existence of $10 \%$ uncertainty when $K_{1}=20$ and $K_{2}=0.002 . x_{1}(t)$ : Tumor volume, $x_{2}(t)$ : Supporting vasculature volume, $u(t)$ : Inhibitory agent rate 

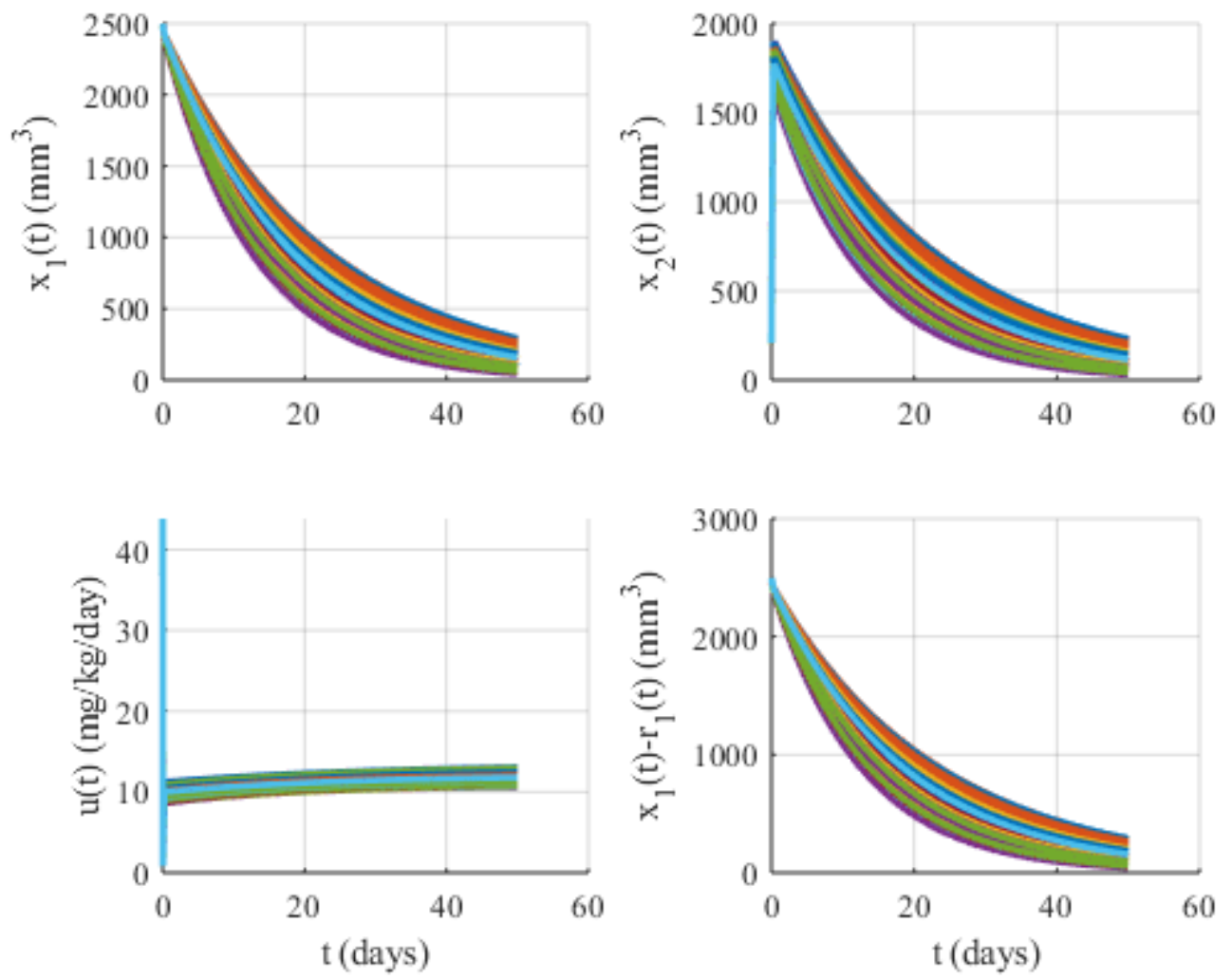

Figure 7: Simulations under the existence of $10 \%$ uncertainty when $K_{1}=20$ and $K_{2}=0.01 . x_{1}(t)$ : Tumor volume, $x_{2}(t)$ : Supporting vasculature volume, $u(t)$ : Inhibitory agent rate 

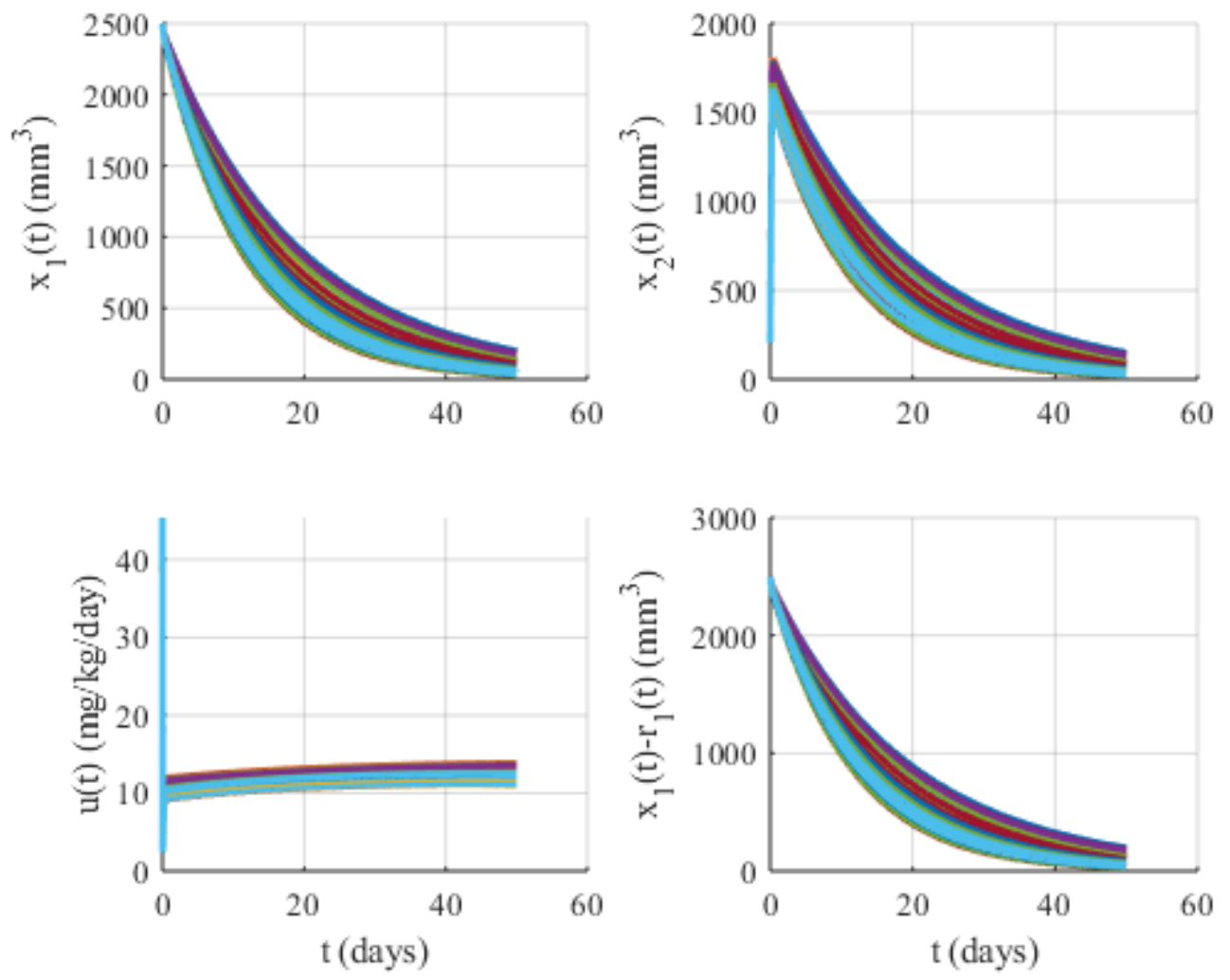

Figure 8: Simulations under the existence of $10 \%$ uncertainty when $K_{1}=20$ and $K_{2}=0.02 . x_{1}(t)$ : Tumor volume, $x_{2}(t)$ : Supporting vasculature volume, $u(t)$ : Inhibitory agent rate 

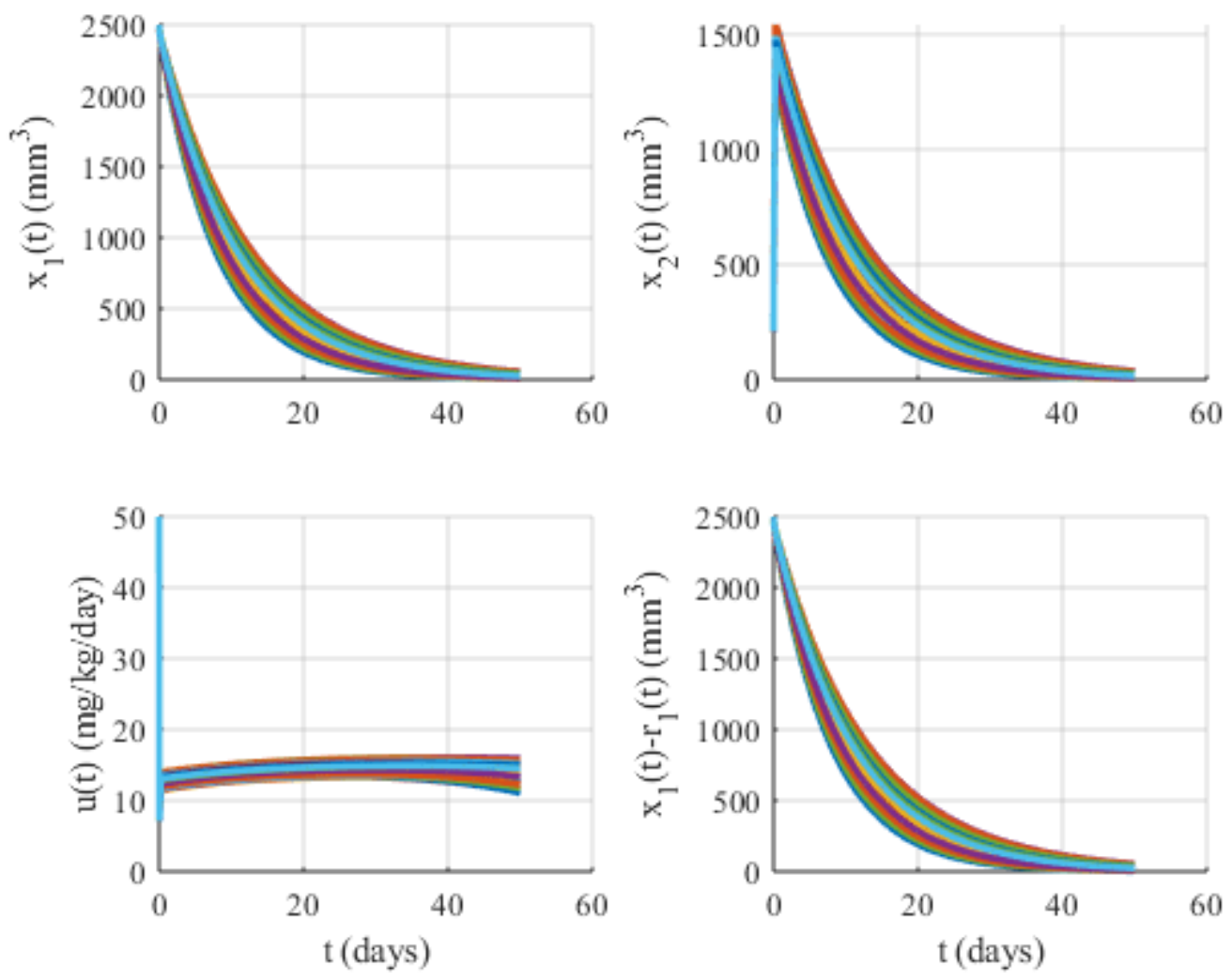

Figure 9: Simulations under the existence of $10 \%$ uncertainty when $K_{1}=20$ and $K_{2}=0.05 . x_{1}(t)$ : Tumor volume, $x_{2}(t)$ : Supporting vasculature volume, $u(t)$ : Inhibitory agent rate 

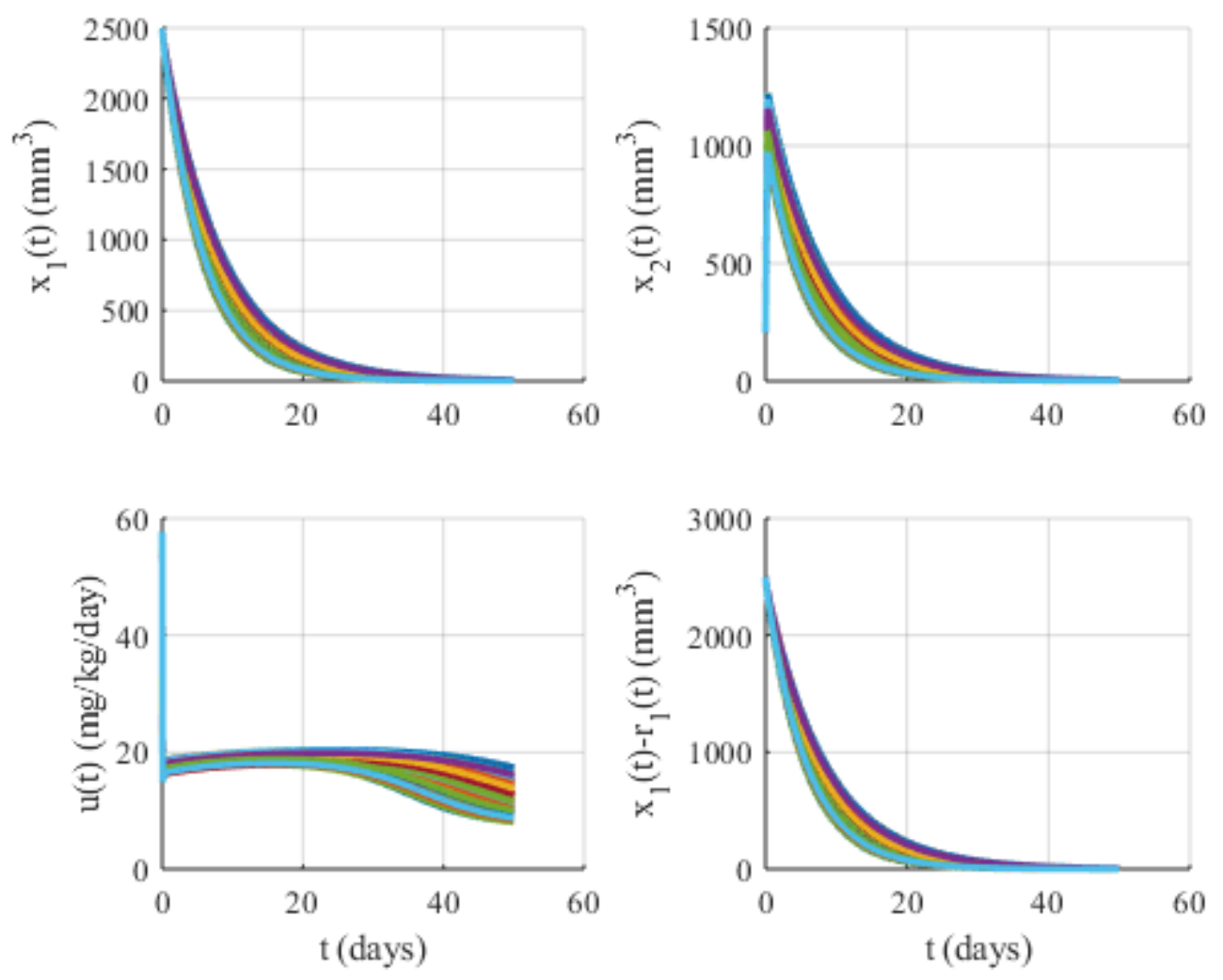

Figure 10: Simulations under the existence of $10 \%$ uncertainty when $K_{1}=20$ and $K_{2}=0.1 . x_{1}(t)$ : Tumor volume, $x_{2}(t)$ : Supporting vasculature volume, $u(t)$ : Inhibitory agent rate 

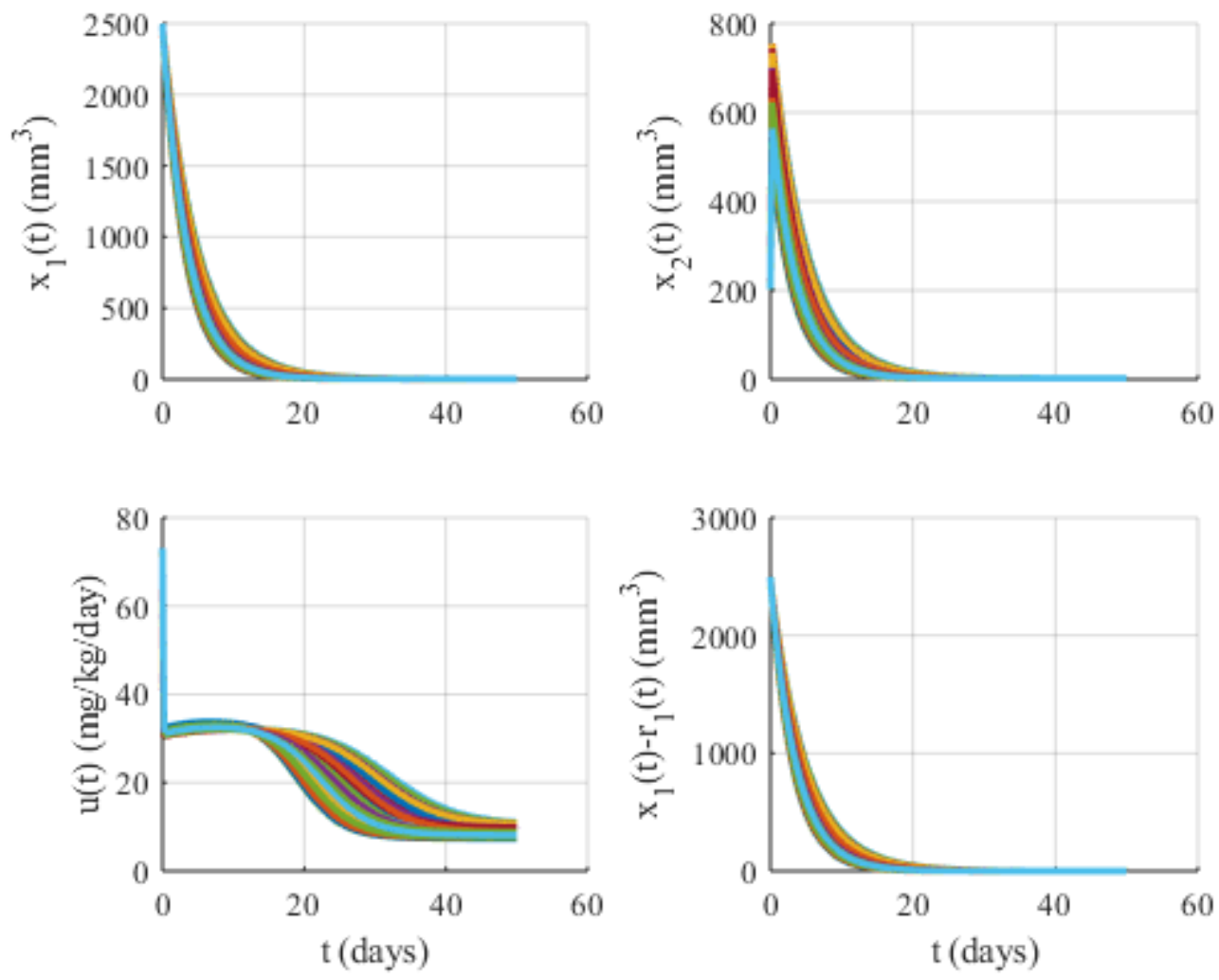

Figure 11: Simulations under the existence of $10 \%$ uncertainty when $K_{1}=20$ and $K_{2}=0.2 . x_{1}(t)$ : Tumor volume, $x_{2}(t)$ : Supporting vasculature volume, $u(t)$ : Inhibitory agent rate 

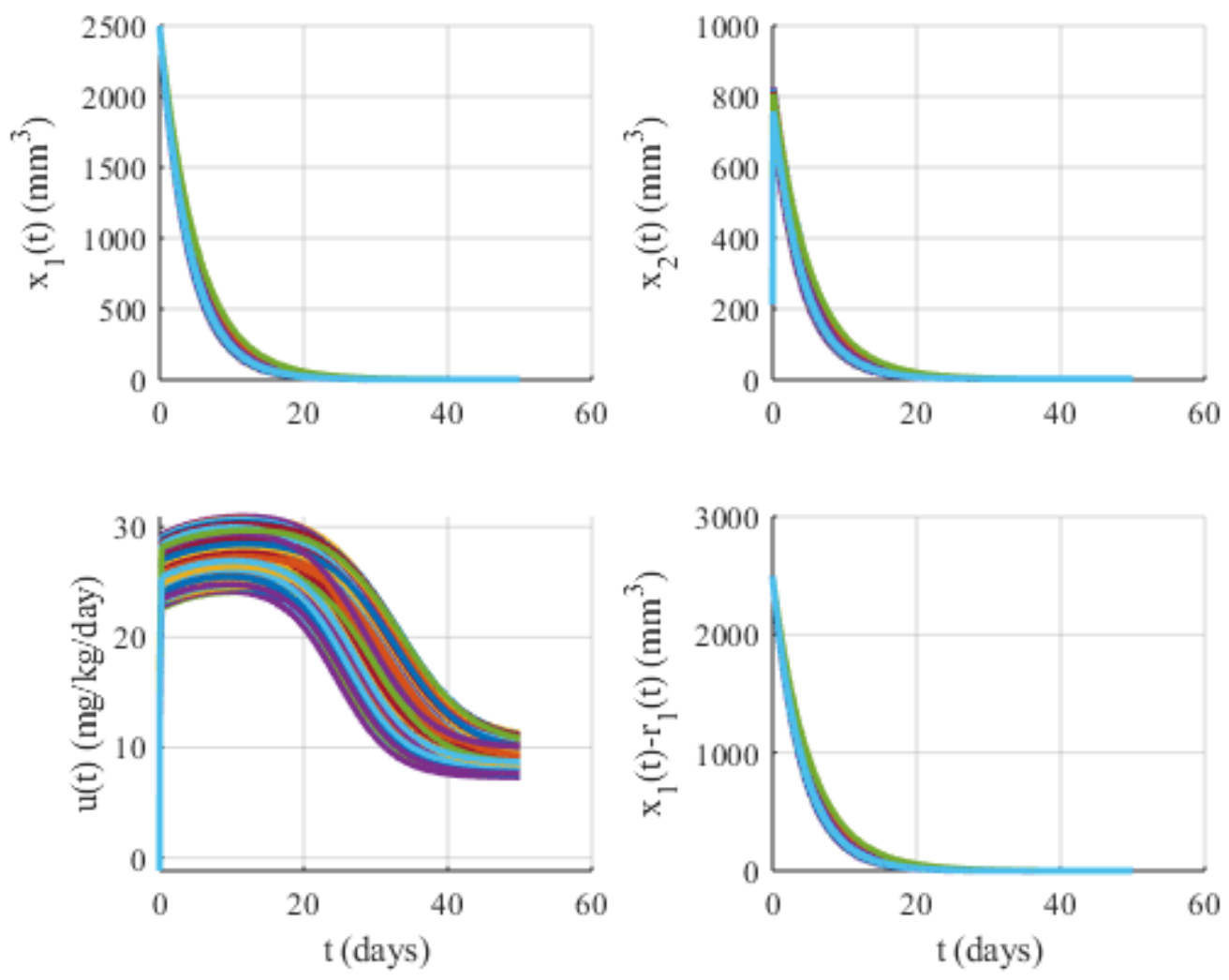

Figure 12: Simulations under the existence of $10 \%$ uncertainty when $K_{1}=50$ and $K_{2}=0.2 . x_{1}(t)$ : Tumor volume, $x_{2}(t)$ : Supporting vasculature volume, $u(t)$ : Inhibitory agent rate 

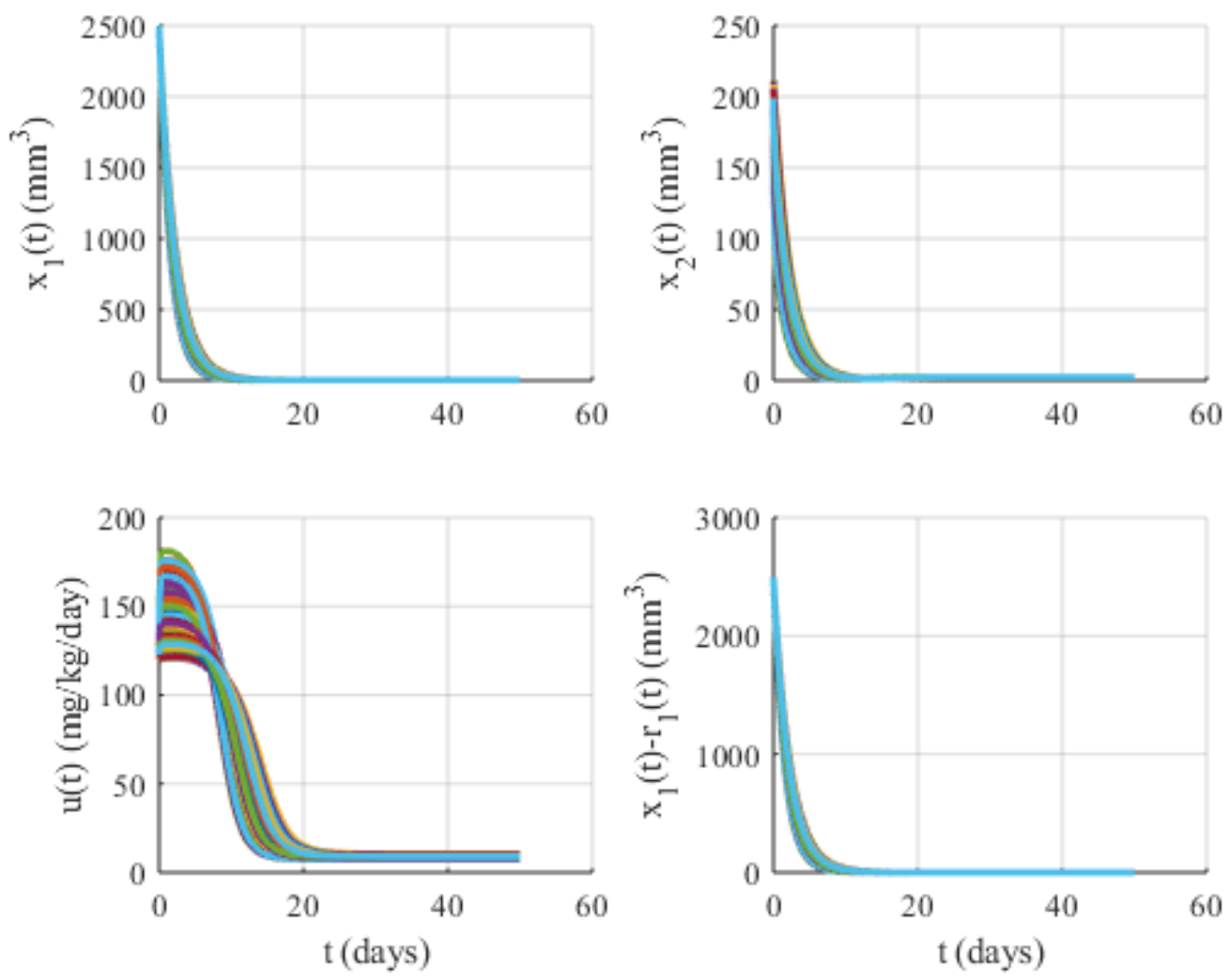

Figure 13: Simulations under the existence of $10 \%$ uncertainty when $K_{1}=50$ and $K_{2}=0.5$. $x_{1}(t)$ : Tumor volume, $x_{2}(t)$ : Supporting vasculature volume, $u(t)$ : Inhibitory agent rate 

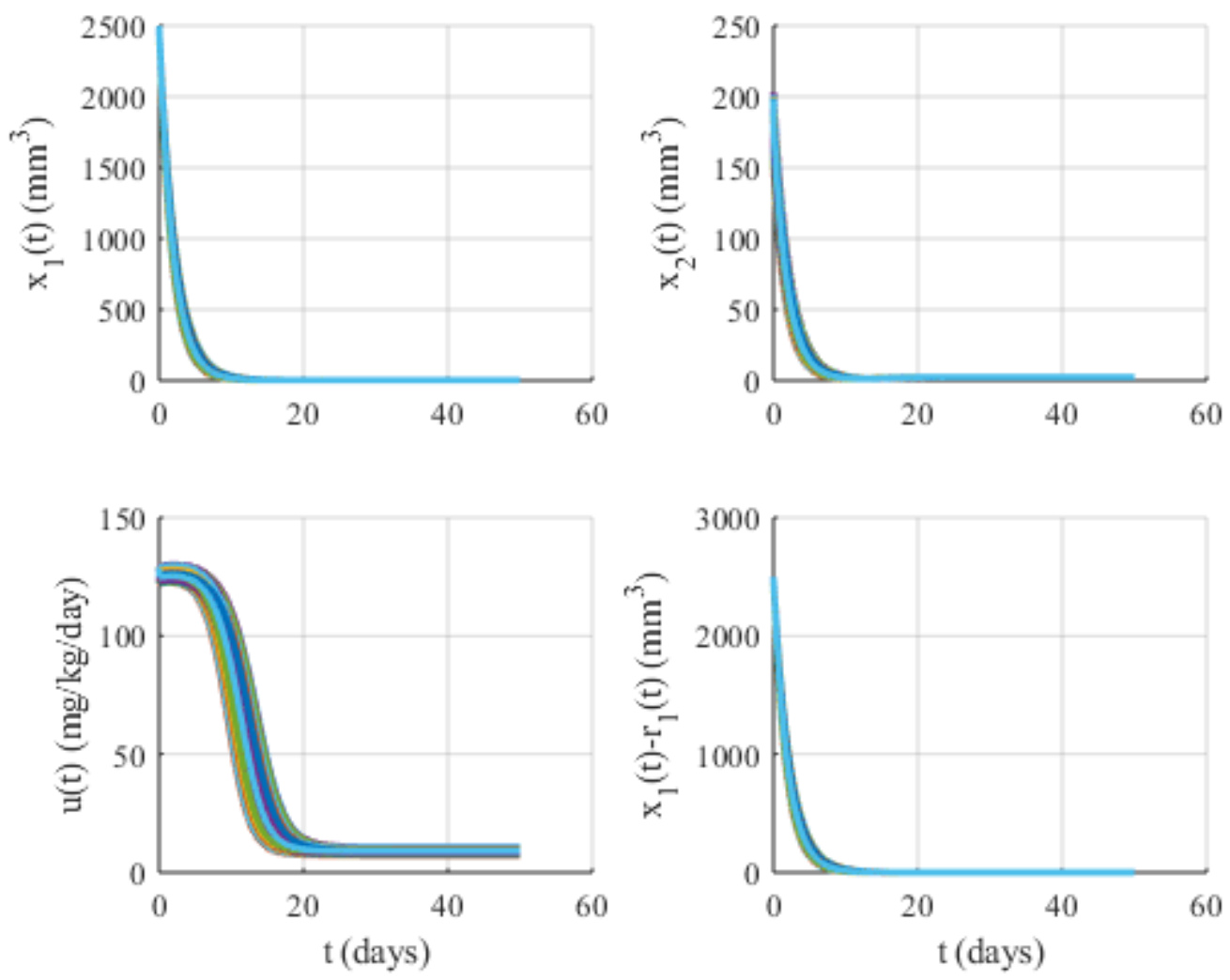

Figure 14: Simulations under the existence of $10 \%$ uncertainty when $K_{1}=100$ and $K_{2}=0.5$. $x_{1}(t)$ : Tumor volume, $x_{2}(t)$ : Supporting vasculature volume, $u(t)$ : Inhibitory agent rate 

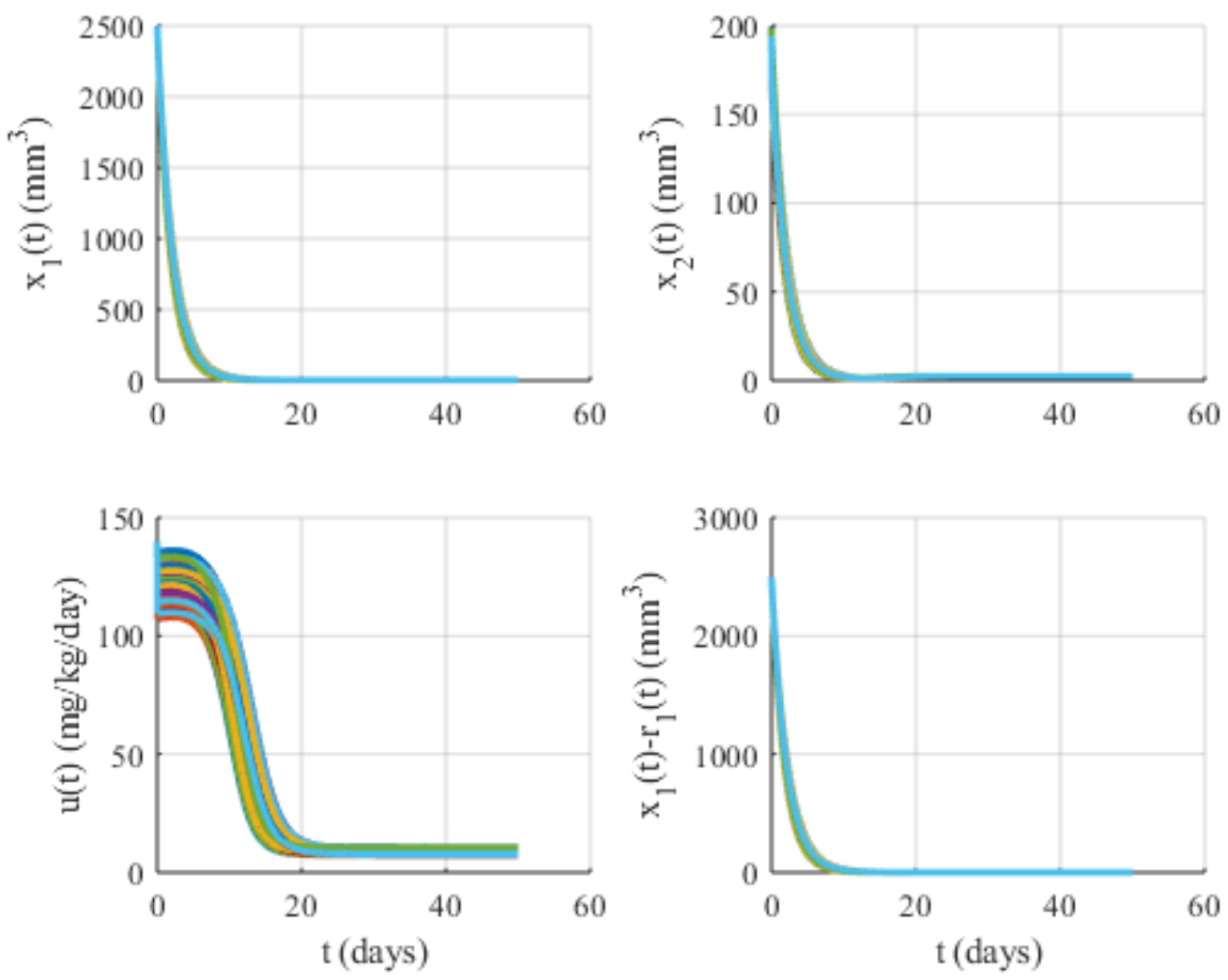

Figure 15: Simulations under the existence of $10 \%$ uncertainty when $K_{1}=200$ and $K_{2}=0.5 . x_{1}(t)$ : Tumor volume, $x_{2}(t)$ : Supporting vasculature volume, $u(t)$ : Inhibitory agent rate 

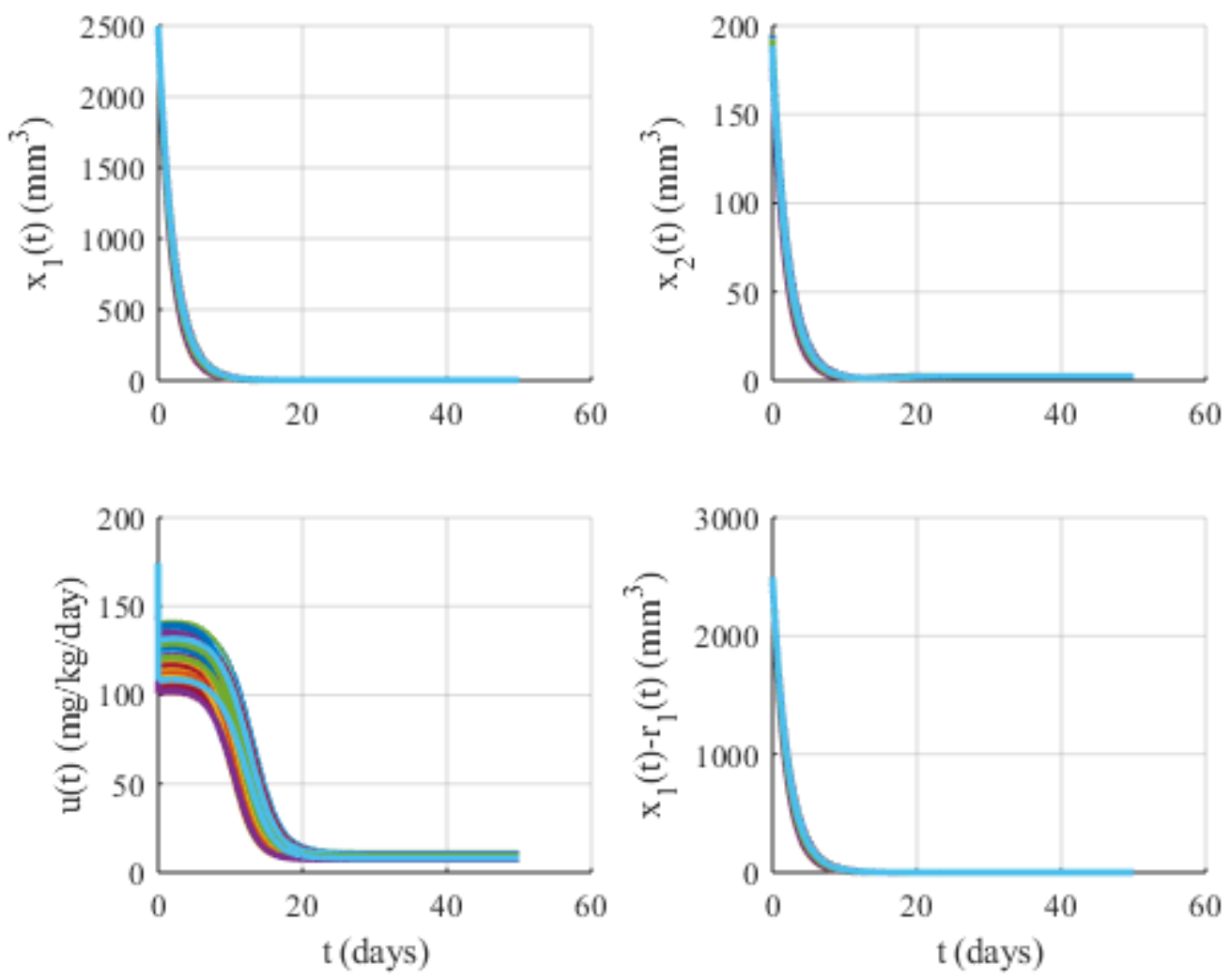

Figure 16: Simulations under the existence of $10 \%$ uncertainty when $K_{1}=500$ and $K_{2}=0.5$. $x_{1}(t)$ : Tumor volume, $x_{2}(t)$ : Supporting vasculature volume, $u(t)$ : Inhibitory agent rate

\section{Results, Discussion and Observation}

In this text, we presented an automatically controlled angiogenic inhibition problem where the control laws are derived recursively using a back-stepping based approach. The mechanism samples the tumor and supporting vasculature volumes and generates an inhibitory agent variation profile. One can have the following observations:

1. Among the cases in Table 4, the most feasible configuration seems the one with $K_{1}=100$ and $K_{2}=0.5$. The variations of the variables are relatively faster, smooth and lowest dispersion seen under various parametric deviation. The setup time seems 9.23 days and highest level of inhibitory agent injection is $128.4 \mathrm{mg} / \mathrm{kg}$.day. See Figure $\mathbf{1 4}$

2. Most of the cases in Table 4 yields sudden decreases in the inhibitory agent administration rate $u(t)$ and jumps in the supporting vasculature volume $x_{2}(t)$. 
3. A robust control approach with better uncertainty accommodation such as sliding mode may be preferred.

\section{References}

[1] P. Hahnfeldt, D. Panigrahy, J. Folkman, L. Hlatky, Tumor development under angiogenic signaling: a dynamical theory of tumor growth, treatment response, and postvascular dormancy, Cancer research 59 (19) (1999) 4770-4775.

[2] A. Szeles, D. A. Drexler, J. Sápi, I. Harmati, L. Kovács, Model-based angiogenic inhibition of tumor growth using adaptive fuzzy techniques, Periodica Polytechnica Electrical Engineering and Computer Science 58 (1) (2014) 29-36.

[3] B. Rashidi, M. Yang, P. Jiang, E. Baranov, Z. An, X. Wang, A. Moossa, R. Hoffman, A highly metastatic lewis lung carcinoma orthotopic green fluorescent protein model, Clinical \& experimental metastasis 18 (1) (2000) 57-60.

[4] S. Herdjunanto, Simple control design using double feedback structure for reducing tumor size, in: Proceedings of 2016 1st International Conference on Biomedical Engineering: Empowering Biomedical Technology for Better Future, IBIOMED 2016, 2017.

URL www . scopus. com

[5] P. Kokotović, M. Arcak, Constructive nonlinear control: a historical perspective, Automatica 37 (5) (2001) 637-662. 